\title{
BACIGALUPO Ana Mariella, Shamans of the Foye Tree: gender, power, and healing among Chilean Mapuche
}

\section{Sabine Kradolfer}

\section{OpenEdition}

Journals

Édition électronique

URL : https://journals.openedition.org/jsa/10819

DOI : 10.4000/jsa. 10819

ISSN : 1957-7842

Éditeur

Société des américanistes

Édition imprimée

Date de publication : 23 juillet 2009

Pagination : 247-250

ISSN : 0037-9174

\section{Référence électronique}

Sabine Kradolfer, «BAcigalupo Ana Mariella, Shamans of the Foye Tree: gender, power, and healing

among Chilean Mapuche », Journal de la Société des américanistes [En ligne], 95-1 | 2009, mis en ligne le 10 juillet 2009, consulté le 04 septembre 2022. URL : http://journals.openedition.org/jsa/10819 ; DOI : https://doi.org/10.4000/jsa.10819

Ce document a été généré automatiquement le 4 septembre 2022

Tous droits réservés 


\title{
BACIGALUPO Ana Mariella, Shamans of the Foye Tree: gender, power, and healing among Chilean Mapuche
}

\author{
Sabine Kradolfer
}

\section{RÉFÉRENCE}

BACIGALUPO Ana Mariella, Shamans of the Foye Tree: gender, power, and healing among Chilean Mapuche, University of Texas Press, Austin, 2007, xi + 321 p., bibl., index, gloss., photos, ill., cartes

1 Le titre principal de l'ouvrage Shamans of the Foye Tree est bien plus qu'une simple paraphrase pour définir les machi (chamanes) mapuche qui utilisent les feuilles et les branches de leur arbre de vie, le foye (Drimys winteri), connu sous le nom de canelo en espagnol, dans nombre de leurs rituels pour mettre les mondes de la nature, des hommes et des esprits en contact. Comme nous l'indique Ana Mariella Bacigalupo, le foye et ses fleurs hermaphrodites entrent en résonance avec le travestissement rituel des machi hommes et en renforcent la légitimité. Cet éclaircissement qui apparaît rapidement dans les premières pages de l'introduction résume la problématique centrale de l'ouvrage contenue dans son sous-titre Gender, power and healing among Chilean Mapuche. C'est en effet de genre et de pouvoir dont il sera question au long des différents chapitres dans lesquels l'auteur poursuit l'objectif qu'elle s'est assigné depuis plusieurs années : faire comprendre à la société chilienne, machiste et dominante, que le travestissement des machi hommes ne peut, et ne doit, pas être compris comme une pratique homosexuelle transgressive, mais comme l'inscription de ces hommes dans des pratiques co-genrées. Ce terme est emprunté à Barbara Tedlock ${ }^{1}$ qui l'utilise pour se référer à des personnalités en partie féminines et en partie masculines. Ce n'est que dans cette position d'articulation de deux genres que les machi peuvent exercer leur art et communiquer avec les esprits, invoquer les forces féminines et masculines, jeunes et âgées qui forment 
les quatre composantes de la divinité mapuche Ngünechen (le Vieil Homme, la Vieille Femme, le Jeune Homme, la Jeune Femme).

En 1991, au début de ses recherches, Ana Mariella Bacigalupo avait été frappée par le pouvoir spirituel que les machi (qui sont soit des femmes, soit des hommes qui se travestissent pour exercer leur art) détiennent au sein de la société mapuche patrilinéaire et patrilocale. Après avoir tenté, dans un premier temps, d'identifier des différences entre les pratiques des femmes et des hommes machi, elle dut cependant se rendre à l'évidence que l'influence du sexe et des rôles de femmes ou d'hommes des machi dans la vie quotidienne est marginale. Dans Shamans of the Foye Tree, après plus de quinze années de recherche, l'auteur nous livre une analyse exhaustive du chamanisme mapuche dans laquelle elle montre la fluidité et la complexité des relations de genre qui ont parfois été négligées par les anthropologues et renvoyées à l'homosexualité, au folklore et à la sorcellerie par la société chilienne, majoritaire et dominante. Les machi et les Mapuche répondent à ces catégorisations stigmatisantes en se les réappropriant et en les transformant pour offrir de nouvelles interprétations de la sorcellerie, de la sexualité et de la thérapie. Afin de rendre leurs identités co-genrées plus adéquates au système de genre de la société chilienne, les machi hommes cherchent à se masculiniser en s'inscrivant dans des positions sociales valorisées au sein de cette société, telles que celles de médecins, de curés ou de guerriers spirituels. Les machi femmes doivent, quant à elles, articuler leurs rôles de filles, d'épouses, de mères avec leurs fonctions religieuses qui les éloignent régulièrement de leur domicile et les détournent de leurs obligations familiales, ce qui est souvent interprété, tant par les Mapuche que par les non-Mapuche, comme des attitudes masculines.

3 Puisque les machi traitent avec des esprits féminins et masculins et qu'elles et ils intègrent aussi bien des symboles et des rôles féminins que masculins dans leurs pratiques chamaniques, leurs attitudes co-genrées remettent en question l'association ordinaire du sexe biologique avec des comportements genrés. De plus, selon les contextes dans lesquels elles et ils s'expriment (vie quotidienne, activités rituelles ou sphère politique), les machi occupent des positions multiples au sein de différents systèmes de genre. Cette situation ambiguë permet aux machi de marquer des frontières ou de créer des ponts entre les idéologies locale (mapuche) et nationale (chilienne) ; bref, elle fonctionne comme marqueur identitaire.

4 À partir de la question du travestisme rituel comme pratique co-genrée, l'auteur nous invite, au long des neuf chapitres qui forment l'ouvrage, à nous intéresser au chamanisme mapuche en l'abordant à partir d'une analyse où les questions du genre, de la notion de personne, des hiérarchies sociales, de la sexualité, des relations de pouvoir, des états altérés de conscience, etc. sont abordées de manière novatrice et détaillée, tant du point de vue des Mapuche que de celui de la société chilienne dominante, présente et passée. Après un premier chapitre introductif, l'auteur présente l'ambiguïté de la position des machi (chapitre 2) qui, comme les sorcier(e)s, sont en contact avec des forces spirituelles, utilisent des instruments et des substances similaires et mobilisent un même univers symbolique, que ce soit, respectivement, pour soigner ou pour nuire. La ligne de séparation entre les machi et les sorcier(e)s est, de ce fait, ténue. Les six chapitres suivants peuvent être organisés en paires qui abordent les identités rituelles de genre des machi en relation avec les pouvoirs cosmiques, la colonisation et les contextes sociaux contemporains (chapitres 3 et 4), la représentation des identités de genre et la sexualité dans les discours coloniaux et nationaux (chapitres 5 et 6 ) et la manière dont les machi 
hommes et femmes négocient les attentes genrées des esprits avec celles des Mapuche et celles de la société chilienne dans leur vie quotidienne, leurs pratiques thérapeutiques et leurs actions politiques (chapitres 7 et 8). Au fil de la lecture, on découvre ainsi non seulement les relations que les machi entretiennent avec des esprits ou des animaux, la symbolique de l'unité qui préside à l'organisation du cosmos, mais aussi comment la situation coloniale a modifié le système de genre mapuche, totalement incompréhensible pour les soldats espagnols et les prêtres jésuites, obligeant aujourd'hui encore les machi à négocier leurs identités de genre en s'adaptant tout en résistant à l'idéologie homophobe de l'État chilien. L'auteur nous montre aussi comment les machi ont intégré des éléments de la modernité, puisqu'elles et ils soignent le stress et la dépression, et qu'elles et ils sont des symboles des luttes pour la récupération des territoires autochtones. La portée théorique de ses analyses ainsi que les réflexions méthodologiques, déontologiques et éthiques sur le rôle de l'anthropologie et de l'anthropologue qu'elle nous livre en introduction (chapitre 1) et en conclusion (chapitre 9) ouvrent nombre de pistes de réflexion et nous invitent à nous interroger quant à nos pratiques et méthodes de recherche.

Du côté des points faibles de cet ouvrage, on regrettera peut-être le peu d'informations fournies par l'auteur, notamment dans le chapitre 6, quant aux questions de définition de la communauté mapuche, de son organisation sociale, des relations de parenté, de la territorialité et des conflits inter-ethniques entre Mapuche et Chiliens non mapuche. Ana Mariella Bacigalupo fait cependant référence et répond dans le chapitre 9 à une critique véhémente allant dans le même sens, qui lui avait été adressée par Guillaume Boccara à l'occasion de la publication d'un précédent ouvrage ${ }^{2}$, critique à laquelle elle avait par ailleurs répondu ${ }^{3}$. Son objectif n'est pas de brosser un tableau de la société mapuche à partir de l'étude du chamanisme, mais bien de restituer une polyphonie de voix et de regards portés par et sur les machi pour contribuer à la compréhension d'un phénomène particulier, tant du point de vue emic qu'etic.

6 Finalement, il faut saluer la qualité et le détail de l'ethnographie des pratiques chamaniques - alors que les Mapuche sont relativement peu enclins à communiquer des informations relevant de leur religiosité et de leurs croyances - rendus possibles par la relation de confiance que l'auteur a pu établir avec ses informateurs et ses informatrices au fil des ans. Pour la rédaction de Shamans of the Foye Tree, elle s'appuie sur des données recueillies entre 1991 et 2006 et, notamment, sur les récits et les expériences de six machi : trois femmes et trois hommes desquel(le)s elle est particulièrement proche. Ana Mariella Bacigalupo restitue ainsi, dans une approche compréhensive, des descriptions très détaillées et très fouillées de situations qu'elle a pu observer et auxquelles elle a parfois participé activement puisqu'elle a été l'assistante d'une machi (cette expérience est relatée dans le chapitre 2) et qu'elle les a consulté(e)s à différentes occasions pour des maux divers. Outre l'analyse de sa propre subjectivité à travers un regard réflexif porté sur ses expériences de recherche, elle étaye ses propos en s'appuyant sur des entretiens avec de nombreux(ses) machi, ainsi que sur des recherches d'archives et documentaires.

7 La qualité et la finesse des observations, alliées à la pertinence des analyses, font de cet ouvrage, écrit dans un style très plaisant et illustré de nombreuses photographies prises par l'auteur, une ethnographie du chamanisme mapuche et des relations de genre qui force à la réflexion et à la déconstruction de nos catégories d'analyse. 


\section{NOTES}

1. Barbara Tedlock, "Recognizing and celebrating the feminine in shamanic heritage ", in Mihaly Hoppal (éd.), Rediscovery of shamanic heritage, Akademiai Kiado, Budapest, 2003, pp. 297-316.

2. Guillaume Boccara, Compte-rendu d'« Ana Mariella Bacigalupo, La Voz del Kultrun en la modernidad. Tradición y cambio en la terapéutica de siete machi mapuche, Ediciones Universidad Católica de Chile, Santiago, 2001, 273 p.», L'Homme, 166, 2003 [http://lhomme.revues.org/ document18713.html].

3. Ana Mariella Bacigalupo, "Local Shamanic knowledges. A response to Guillaume Boccara ", L'Homme, 169, 2004, pp. 219-224.

\section{AUTEURS}

\section{SABINE KRADOLFER}

Faculté des SSP, Université de Lausanne (Suisse) 